Review

\title{
Up-Conversion Nanoparticles for Gastric Cancer Targeted Imaging and Therapy
}

\author{
Yuming Yang ${ }^{1,2}$, Yu Han ${ }^{1,2}$, Caixia Yue ${ }^{1,2}$ \\ ${ }^{1}$ Department of Instrument Science and Engineering, School of Electronic Information and Electrical Engineering, Shanghai Jiao \\ Tong University, Shanghai 200240, China. \\ ${ }^{2}$ Shanghai Engineering Research Center for Intelligent Diagnosis and Treatment Instrument, Shanghai 200240, China. \\ $\triangle$ Corresponding author. E-mail: yumingyang@sjtu.edu.cn
}

Received: Sep. 18, 2016; Accepted: Sep. 20, 2016; Published: Sep. 26, 2016.

Citation: Yuming Yang, Yu Han and Caixia Yue, Up-Conversion Nanoparticles for Gastric Cancer Targeted Imaging and Therapy. Nano Biomed. Eng., 20|6, 8(3): $|6|-|7|$.

DOI: 10.5101/nbe.v8i3.p161-171.

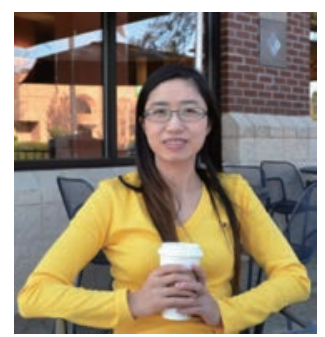

Yuming Yang was born in 1981 in Changchun, China. In 2009, she received her Ph.D. degree from Jilin University, and went to Fudan University as postdoctoral fellow. In order to enhance research level, Dr. Yang further made research cooperation with the department of Regenerative Medicine and Cell Biology, Medical University of South Carolina, USA and took office in University of Louisville in USA as a postdoctoral researcher during the years of 2012-2014. Since January 2015, she joined the School of Electronic Information and Electrical Engineering, Shanghai Jiao Tong University. Her major research interest is design and synthesis of multifunctional materials for tumor imaging and therapy.

\begin{abstract}
As a new generation of fluorophores, up-conversion nanoparticles (UCNPs) exhibit high penetration depth in tissues, low photodamage to biological samples, large Stokes shifts, sharp emission bands, free auto-fluorescence background and high photostability, thus making them an ideal kind of fluorescent labels for a variety of assay formats ranging from bio-imaging to cancer therapy. In this article, the method for the synthesis of biocompatible UCNPs, the recent progress regarding the UCNPs for multimodal targeted tumor imaging, drug delivery, photodynamic therapy (PDT), and photothermal therapy (PTT) are discussed on emphasis. The challenges and opportunities in this emerging field are also presented.
\end{abstract}

Keywords: Upconversion; Nanoparticle; Cancer therapy; Imaging; Drug delivery; Detection

\section{Introduction}

One of the most important achievements for the research of gastric cancer is the proposal of early gastric cancer (EGC) concept in 1962. The proposal of early gastric cancer concept aimed to make the clinical cure and longer survival time possible. After half a century of research and diagnosis, the knowledge on early gastric cancer is also gradually deepend. The diagnosis of early gastric cancer can be gradually refined. Especially in recent years, the guiding ideology for early treatment of gastric cancer, the 
original pursuit of longer survival period has changed to more requirements in the minimally invasive surgery to ensure that patients have a good quality of life and so the treatment is also more personalized. At present, the diagnosis rate of early gastric cancer is still less than $10 \%$, while in Japan it is as high as $70 \% \sim 50 \%$. At present, the most important thing is the changing of diagnosis concept and the improvement of diagnosis rate of the early gastric cancer.

To date, much effort has been put on the design of novel upconversion nanophosphors. Surface functionalized and multifunctional UCNPs have been synthesized. Great advances on the improvement of probe targeting, pharmacokinetics, biocompatibility, photophysics, and the maturation of multimodal techniques are also made. In this paper, we will introduce the synthesis, and the toxicity of the UCNPs. The progress of UCNPs applications on biological imaging, cancer, especially gastric cancer in recent years will also be introduced in detail.

\section{Synthesis of Rare Earth Doped UCNPs}

Due to the excellent properties and wide application
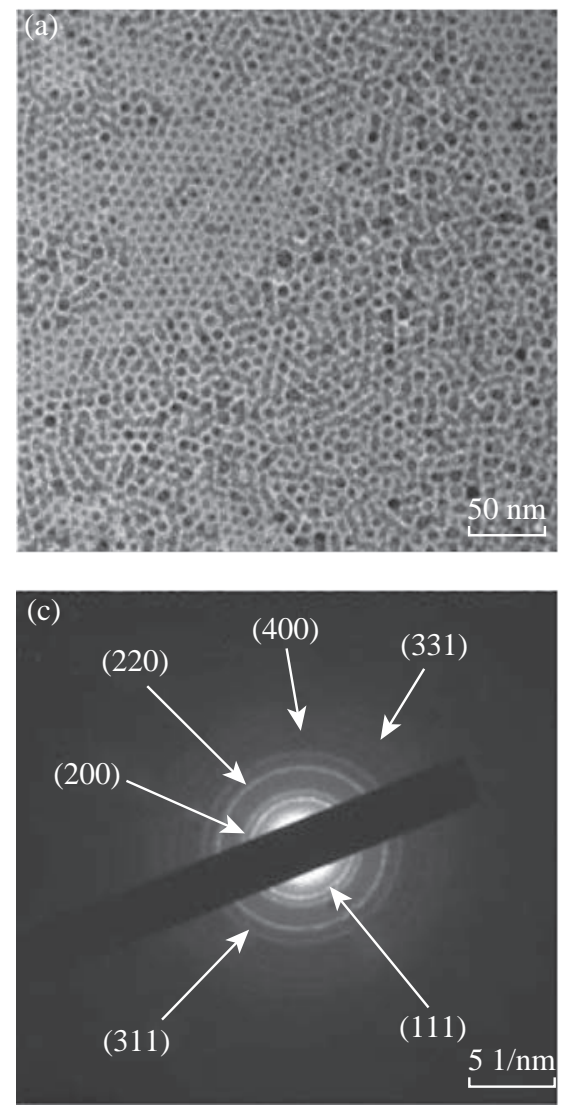

prospect of UCNPs, the development of rare earth has attracted wide attention. At present, different methods have been developed to get upconversion nanophosphors, such as sol-gel [1-9], hydrothermal [10-15], co-precipitation [16-19], and thermal decomposition methods [20-33] etc. Nanoparticles synthesized by sol-gel method are usually not suitable for biological applications due to the particle agglomeration in the process of high temperature treatment. In contrast, hydrothermal method is a good method for the preparation of UCNPs with high crystallinity, homogeneous size, and good dispersion, but the disadvantage is that the process of crystal growth can not be observed. The thermal decomposition method and co precipitation method are developed in recent years, which can synthesize UCNPs with small and homogeneous size. At present, many papers have been reported based on both mehtods. Such as Daxiang Cui's team of Shanghai Jiaotong University synthesized $\mathrm{NaGdF}_{4}: 20 \% \mathrm{Yb}$, $2 \%$ Er based on the thermal decomposition method. The synthesized nanoparticles have good water solubility the $\mathrm{CT}$ imaging and near infrared fluorescence imaging are successfully achieved [34] (see Fig. 1).
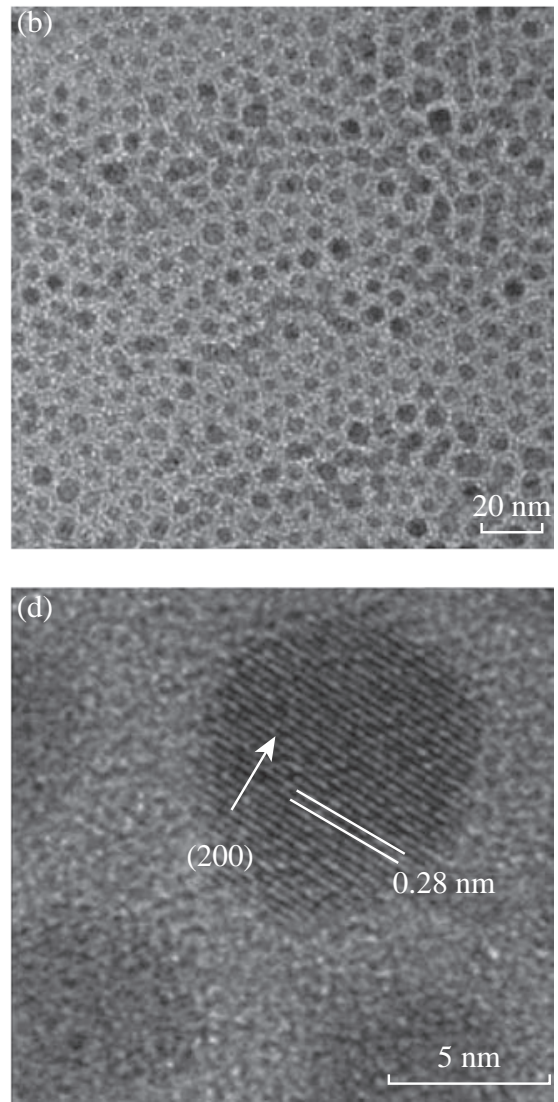

Fig. 1 (a) and (b) TEM images of $\mathrm{NaGdF}_{4}: 20 \% \mathrm{Yb}, 2 \% \mathrm{Er}$; (c) SAED of UCNPs; (d) HR-TEM of NaGdF $: 20 \% \mathrm{Yb}, 2 \%$ Er. Reprinted with permission of Ref. [34]. 
In order to obtain the UCNPs with good biocompatibility, "one step" method was developed in recent years with the help of hydrophilic or binary cooperative ligands, such as polyols [10, 35, 36], EDTA [37-40], citrate [37, 41-45], sodium dodecyl sulfate (SDS) [46], PVP [47], small-molecule binary acid [48, 49], polyethylene glycol (PEG) [49], PVP [50], PAA [51, 52], PEI [51], 3-mercaptopropionic acid (3MA) and 6-aminocaproic acid (6AA) [53] etc. This "one step" method has the advantages of high efficiency and could be operated at mild reaction conditions [54, 55]. Daxiang Cui's group reported the UCNPs with different mophorlogy in the presence of n-octanol and 1-butyl-3-methylimidazolium tetrafluoroborate [56]. The results show that the UCNPs synthesized by this method have good water solubility, and that the 1-butyl-3-methylimidazolium tetrafluoroborate palys very important role in controlling the size and morphology of the UCNPs (see Fig. 2) .

\section{UCNPs Used for Gastric Cancer Imaging}

As a new imaging technique, UCNPs imaging offers a unique approach for visualizing morphological details in tissue with sub cellular resolution, and has come to be a powerful noninvasive tool for visualizing the full range of bio-species from living cells to animals. Exploitation in general internalization studies was performed using bare or modified UCNPs. They have been widely used for in vitro or in vivo imaging to demonstrate their promise in biological in vivo applications [57-60]. Kobayashi et al. demonstrated in vivo multiple color lymphatic imaging using upconverting nanocrystals in 2009 [61]. Multicolor in vivo lymph node mapping UCL imaging was further demonstrated by Liu's group in the following year, and found the in vivo detection limit of UCNPs to be at least one order of magnitude lower than that of QDs [62]. Li's group reported a series of articles related the UCNPs for lymphatic imaging [63-65]. Niagara et al. applied silica/ $\mathrm{NaYF}_{4}$ : $\mathrm{Yb}, \mathrm{Er}$ to dynamically track live myoblast cells in vitro and in a living mouse model of cryo-injured hind limb [66]. In vivo confocal imaging of nanoparticle-loaded cells intravenously injected into a mouse tail vein showed them flowing in the ear blood vessels. Nanoparticle-loaded cells were also unambiguously identified with superior contrast
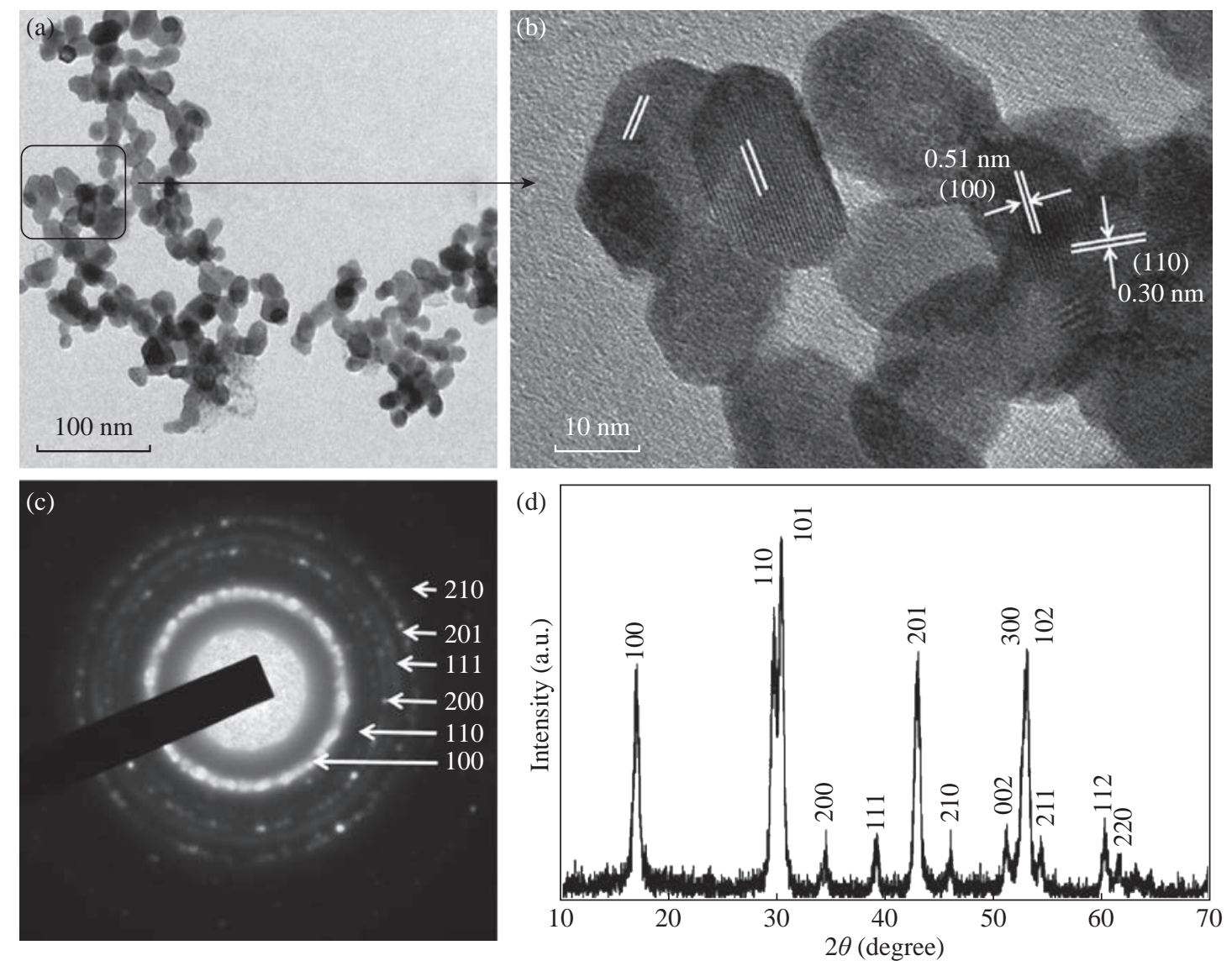

Fig. 2 (a) TEM image of $\mathrm{NaGdF}_{4}$ : Yb, Er (n-octanol $(10 \mathrm{~mL}) / \mathrm{BmimPF}_{6}(15 \mathrm{~mL})$ ); (b) Magnification of selected area; (c) The corresponding SAED pattern; (d) The corresponding XRD pattern. Reprinted with permission of Ref. [56]. 
against a negligible background at least $1[300 \mu \mathrm{m}$ deep in a fully vascularized living tissue upon intramuscular injection.

Tumor targeted molecular imaging plays a very important role in the diagnosis and prognosis of gastric cancer [67]. It has absorbed a wide range of attention in the use of UNCPs for tumor imaging in vivo. Different kinds of antibodies, aptamers, small molecules and peptides were conjugated on the surface of the UCNPs for targeted imaging of specific kinds of cells or biological molecules. Zako originally reported the tumor cell-targeted upconversion imaging using UCNPs modified with cyclic RGD peptide (RGD- $\mathrm{Y}_{2} \mathrm{O}_{3}$ ) [68]. FA is also widely chosen as targeting ligands for specific targeting and imaging of cancer cells. Cui's group reported the $\mathrm{NaYbF}_{4}: 25 \% \mathrm{Gd}, 2 \% \mathrm{Tm} / \mathrm{SiO}_{2}$ modified by FA, which could be applied on the gastric cancer imaging in vivo [69] (Fig. 3).
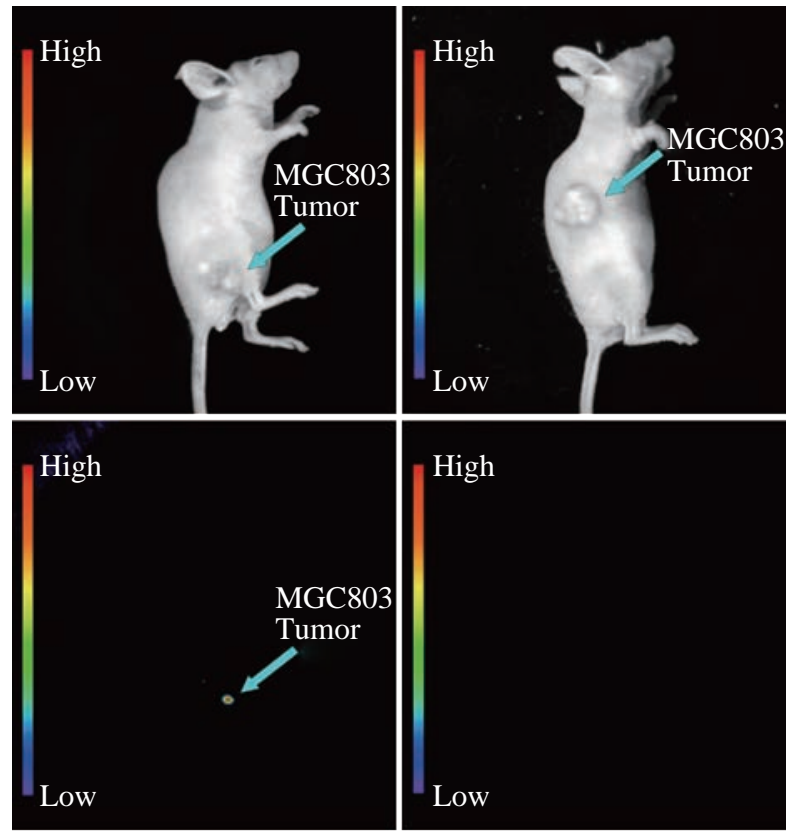

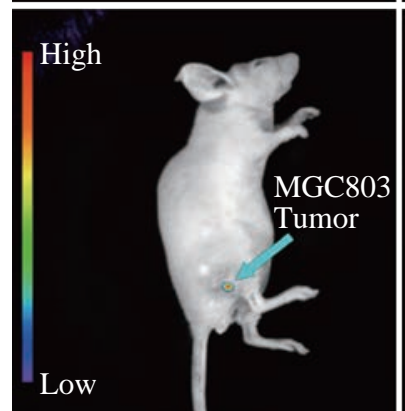

(a)

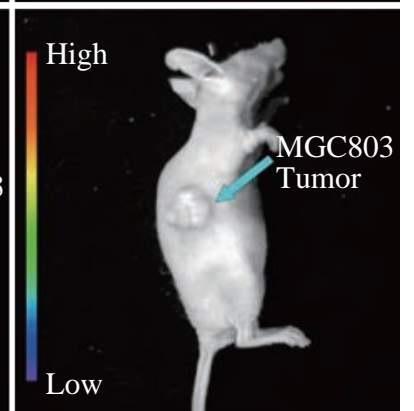

(b)
Fig. 3 Targeted in vivo NIR luminescence imaging of subcutaneous MGC803 tumor (indicated by short arrows) after intravenous injection of (a) $\mathrm{NaYbF}_{4}: 25 \% \mathrm{Gd}, 2 \% \mathrm{Tm}-\mathrm{FA}$; and (b) $\mathrm{NaYbF}_{4}: 25 \% \mathrm{Gd}, 2 \% \mathrm{Tm} @ \mathrm{SiO}_{2}-\mathrm{NH}_{2}$ nanoparticles for $4 \mathrm{~h}$. Reprinted with permission of Ref. [69].
In addition to the optical imaging technique, molecular imaging technique also contains positron emission tomography (PET), magnetic resonance imaging (MRI), Single-Photon Emission Computed Tomography (SPECT) and X-ray computer tomography (CT). These techniques have already become routine clinical examination techniques in hospitals. However, each imaging modality has its own merits and disadvantages, and a single technique does not possess all the required capabilities for comprehensive imaging. Optical imaging as an inexpensive, robust and portable method provides the highest sensitivity and spatial resolution for in vitro imaging, but still lacks the full capability to obtain anatomical and physiological details in vivo. MRI and CT techniques have excellent spatial resolution, good depth for in vivo imaging and exceptional anatomic information, but suffer from limited sensitivity and lack resolution for imaging at the cellular level. $1+\mathrm{HQFH}$ multimodal UCNPs for bioimaging are quickly becoming important tools for biomedical research and clinical diagnostics.

The most commonly used MRI contrast agent is $\mathrm{Gd}^{3+}$ chelate complex. The contrast of MRI imaging is improved by changing the exchange rate of the internal and external layers of the water to shorten the longitudinal relaxation time T1. Prasad group is one of the pioneers who originally developed $\mathrm{Gd}^{3+}$ and $\mathrm{Er}^{3+}$ / $\mathrm{Yb}^{3+} / \mathrm{Eu}^{3+}$ codoped $\mathrm{NaYF}_{4}$ for the dual modality of optical and MR imaging [70]. For UCNPs used as CT contrast agents, there are two approaches to improve the X-ray attenuation coefficient. The first method is to increase the lanthanide elements content in a single particle. Liu et al. prepared the PEGylated $\mathrm{Yb}_{2} \mathrm{O}_{3}$ : Er nanoparticles with high $\mathrm{Yb}$ content in a single particle suitable for both X-ray CT imaging and UCL imaging [71]. The second way is to choose higher atomic number elements among RE elements for the preparation of UCNPs. For the $\mathrm{REF}_{3}$ and $\mathrm{NaREF}_{4}$ fluorides, the La content in $\mathrm{LaF}_{3}$ is $70.9 \%$, much higher than other RE elements content in $\mathrm{NaREF}_{4}$. Therefore, $\mathrm{REF}_{3}$-based UCNPs can serve as excellent CT contrast agents and ideal building blocks for multimodal imaging agents. Based on this mechanism, FA conjugated silica modified $\mathrm{LaF}_{3}$ : Yb,Tm UCNPs (UCNPs@SiO,-FA) with high La content in a single particle were strategically designed by Cui's group for simultaneously targeted dual-modality imaging of UCL and CT [72]. Fig. 4 shows the real-time in vivo UCL images after intravenous injection of UCNPs@ $\mathrm{SiO}_{2}-$ FA in nude mice at $2 \mathrm{~h}$. 


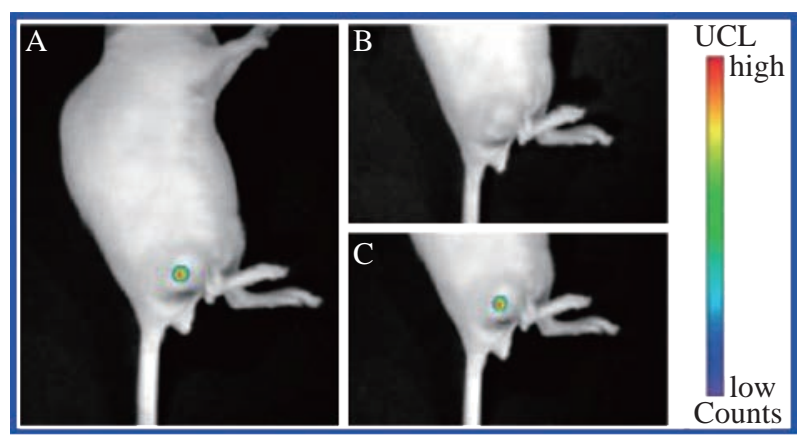

Fig. 4 Real-time in vivo UCL images after intravenous injection of UCNPs@ $\mathrm{SiO}_{2}-\mathrm{FA}$ in nude mice at 2 h. Reprinted with permission of Ref. [72].

$\mathrm{NaGdF}_{4}$ is also a good contrast agent for UCL/CT dual mode imaging [73-77]. Cui's team synthesized rare earth ion doped $\mathrm{NaGdF}_{4}$ micron crystal using one step synthesis method. This material displays good conversion luminescence, magnetism and biocompatibility, and were successfully used for gastric cancer CT imaging [78], as shown in Fig. 5.

Compared with CT scan, an MRI is suited for examining soft tissue, such as ligament and tendon injury, spinal cord injury, brain tumors etc. While a CT scan is better suited for bone injuries, lung and chest imaging, and detecting cancers. Making a combination of UCL, CT and MRI imaging is no doubt a good way to realize better tissue scans [79-82].

PET is a nuclear medical imaging technique that produces a three-dimensional image or picture of functional processes in the body. PET scans are increasingly read alongside CT or MRI scans to provide excellent spatial resolution and high sensitivity. $\mathrm{CT}$ provides exceptional anatomical information, but suffers from limited sensitivity. PET provides a visualization method with high sensitivity, but a low $(\sim \mathrm{mm})$ spatial resolution. However, CT, PET, and MRI are all unsuitable for visualizing living cells because of low planar resolution, but this can be remedied by combining with UCNPs. The combination provides the highest spatial resolution and is suitable for imaging living cells.

\section{UCNPs for Cancer Therapy}

Apart from being used for cancer cell related bioimaging, UCNPs were selected as probes for disease related sensing [83-85] or therapy [86]. For example, mesenchymal stem cells have shown great potential in regenerative medicine. Sensitive and reliable methods for stem cell labeling and in vivo tracking are thus of great importance. Liu's group reported the use of oligoarginine conjugated UCNPs as an exogenous contrast agent to track mouse mesenchymal stem cells in vivo. As few as $\sim 10$ cells labeled with UCNPs are detected in vivo, which highlights the promise of using UCNPs as a new type of ultrasensitive probes for labeling and in vivo tracking of stem cells at nearly the single cell level [87]. In another case, Liu and coworkers developed $\mathrm{MnO}_{2}$ nanosheet modified UCNPs for rapid,
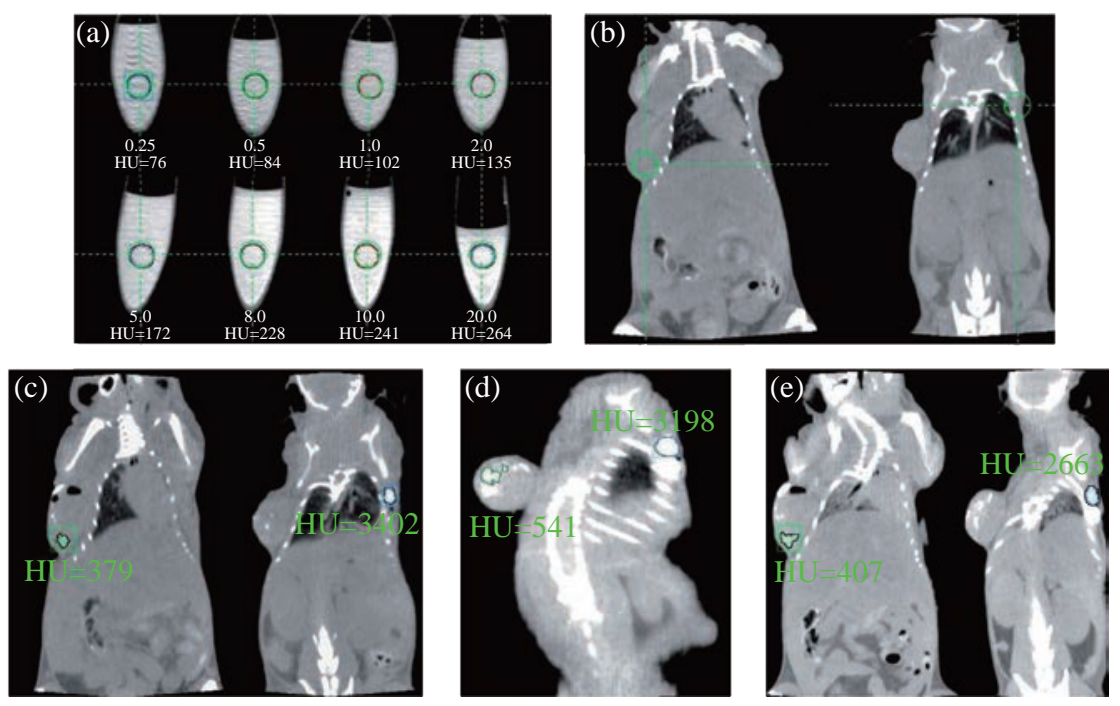

Fig. 5 In vitro CT imaging of (a) $\mathrm{NaGdF}_{4}: \mathrm{Yb}^{3+} / \mathrm{Er}^{3+}$ submicrocrystals suspended in PBS solution; (b) in vivo CT imaging of mouse before injection; (c) after injection; (d) after $2 \mathrm{~h}$ injection; and (e) after $4 \mathrm{~h}$ injection. As for the investigated nude mice, tumor sites are located in the left side of back, and normal tissues are located in the right side of back. Nude mice loaded with gastric cancer MGC803 cells was selected as the animal model. The tumor was implanted and grown in nude mice to an appropriate size (3-5 mm in diameter). Reprinted with permission of Ref. [78]. 
selective detection of glutathione in aqueous solutions and living cells [88].

Up to now, most of the UCNPs drug delivery system were realized by coating PEG or mesoporous silica for probe attachment or drug loading [89-92]. Liu and coworkers functionalized UCNPs with a PEG grafted amphiphilic polymer. Then the PEGylated UCNPs were loaded with DOX molecules and conjugated with FA for targeted drug delivery and cell imaging. The loading and releasing of DOX from UCNPs are controlled by varying $\mathrm{pH}$, with an increased drug dissociation rate in acidic environment, favorable for controlled drug release [89]. Cui and coworkers reported a multifunctional "nanorattle" hollow spheres that consist of RE-doped $\mathrm{NaYF}_{4}$ shells with a $\mathrm{SiO}_{2}$ coated $\mathrm{Fe}_{3} \mathrm{O}_{4}$ inner particle. The material emits visible luminescence upon NIR excitation and can be directed by an external magnetic field to a specific target, making it an attractive system for a variety of biological applications. In vivo experiments exhibit encouraging tumor shrinkage with the DOX loading and significantly enhanced tumor targeting in the presence of an applied magnetic field [93].

Although the $\mathrm{SiO}_{2}$ modified UCNPs have made a great progress, but the core/shell structure needs complicated procedure, and it is difficult to realize batch production. To solve this problem, Cui's group proposed a one-step method for the synthesis of mesoporous structure of UCNPs. Drugs for the treatment of gastric cancer can be filled in the mesoporous of UCNPs and then delivered to the tumor site. SEM images of the synthesized mesoporous UNCPs are shown in Fig. 6.

In view of Magneto-optical imaging features of $\mathrm{NaGdF}_{4}$, the nanoparticles can be successfully applied to CT imaging (Fig. 7) and the delivery of anti tumor drugs for treatment of gastric cancer [94].

Small interfering RNA (siRNA) has emerged as a gene-based therapy due to their highly desirable roles in RNA interference and gene silencing effects in biomedical research. The key requirements for an effective siRNA therapy is that sufficient siRNA needs to be introduced into cells or organs and that the release of siRNA inside target cells in a highly spatial and temporal precision can be remotely controlled. Up to now, a few types of UCNPs have been developed for the purpose of effective siRNA delivery. Zhang and coworkers reported a UCNPs based system for targeted delivery of siRNA to cancer cells. The siRNA was attached to anti-Her2 antibody conjugated UCNPs and the delivery of these nanoparticles to SK-BR-3 cells was studied, which certified the capability of using UCNPs as a fluorescent probe and delivery system for simultaneous imaging and delivery of
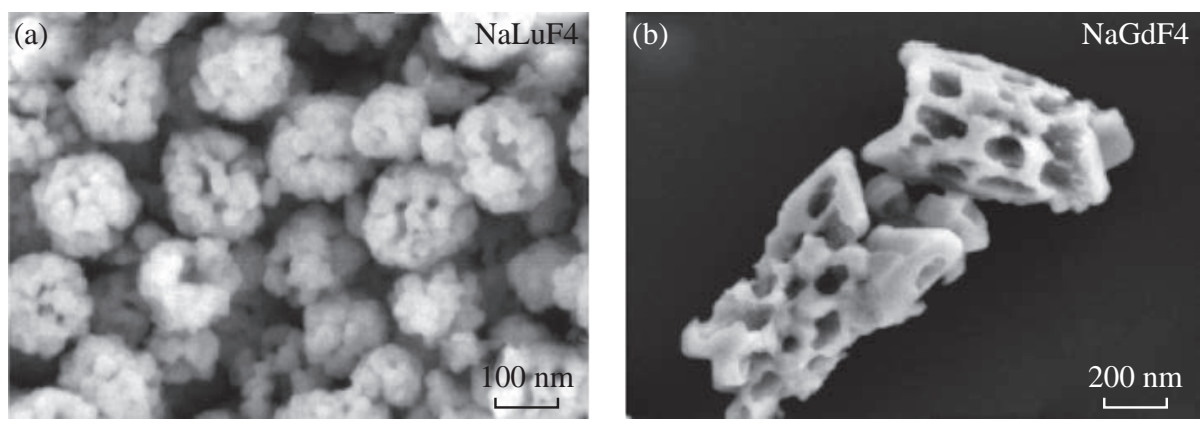

Fig. $6 \mathrm{SEM}$ images of (a) $\mathrm{NaLuF}_{4}$; and (b) $\mathrm{NaGdF}_{4}$.

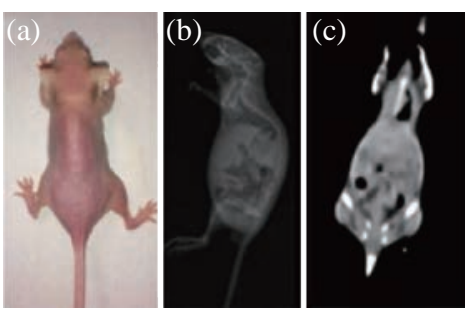

(I) Control group images of vivo nude mice
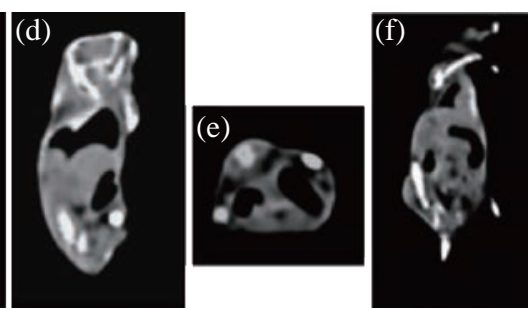

(II) CT images of vivo nude mice

Fig. 7 (I) Images of control group before injection: (a) The photograph of the nude mouse; (b) X-ray image and (c), (d) and (e) CT images of nude mouse model as a control. (II) CT images of (f) coronal plane; (g) sagittal plane; and (h) transverse plane through subcutaneous injection with $\mathrm{NaLuF}_{4}$ upconversion nanoparticles. Reprinted with permission of Ref. [94]. 
biological molecules [95]. Very recently, Yang et al. reported a system of silica coated UCNPs, which were functionalized with cationic photocaged linkers through covalent bonding. Anionic siRNA could be effectively absorbed onto the linkers through electrostatic attractions and were easily internalized by living cells. Upon NIR light irradiation, the photocaged linker on the Si-UCNPs surface could be cleaved by the upconverted UV light and thus initiated the intracellular release of the siRNA [96].

PDT is a relatively new clinical therapeutic modality which involves killing of disease cells by excitation of photosensitizer chemicals with high-energy light to generate reactive oxygen species from surrounding dissolved oxygen [97-99]. However, poor tissue penetration of high-energy light and hydrophobic photosensitizers limits the effectiveness to superficial pathologies. UCNPs can be used to activates the photosensitizers in deep tissue because NIR light can penetrate a few centimeters into soft tissue due to weak absorption in the optical "transparent window". Four main classes of photosensitizers have been approved by the U.S. Food and Drug Administration for clinical use against cancer cells, containing porphyrin derivatives, chlorins, phthalocyanines, and porphycenes [100, 101]. The second-generation photosensitizers of zinc (II)-phthalocyanine ( $\mathrm{ZnPc}$ ) have been proven highly selective for tumor targets and showed enhanced cytotoxic effects both in vitro and in vivo.

PTT employs photo absorbers to generate heat from light absorption, leading to thermal ablation of cancer cells. Various nanomaterials with high NIR light absorbance such as gold and silver nanoshells, nanorods and nanocages etc. have been utilized for PTT treatment of cancer [102]. Song et al. reported the synthesis of core-shell structured hexagonalphase $\mathrm{NaYF}_{4}$ : $\mathrm{Yb}^{3+}, \mathrm{Er}^{3+} @ \mathrm{Ag}$ nanoparticles and their unique biofunctional properties. HepG2 cells fromhuman hepatic cancer and BCap-37 cells from human breast cancer incubated with the composite UCNPs in vitro were found to undergo photothermally induced death on exposure to $980 \mathrm{~nm}$ [103]. Liu and coworkers synthesized a new class of multifunctional nanoparticles consisting a UCNP particle as the core, a layer of ultra-small iron oxide nanoparticles (IONPs) as the intermediate shell, and a thin layer of gold as the outer shell. The layer of IONPs between UCNPs and the Au shell not only affords the magnetic properties but also significantly reduces the luminescence quenching effects of the gold nanostructure to UCNPs. The UCNP-IONP-Au nanoparticles are then coated with PEG to improve its biocompatibility in physiological solutions. Those multifunctional nanoparticles are used for UCL/MR multimodal imaging as well as in vitro photothermal ablation of cancer cells [104]. In the next year of 2012, they achieved highly efficient in vivo magnetically targeted PTT by using the same multifunctional nanoparticles. In vivo dual modal optical/MR imaging of mice uncovers that by placing a magnet nearby the tumor, the UCNPs show high tumor accumulation after intravenous injection, which is $\sim 8$ folds higher than that of without magnetic nearby. An outstanding PTT therapeutic efficacy with $100 \%$ of tumor elimination in a murine breast cancer model is realized [105].

Most recently, Hilderbrand et al. reported the design and synthesis of $\mathrm{NaYF}_{4}: \mathrm{Er}^{3+}, \mathrm{Yb}^{3+} @ \mathrm{SiO}_{2}$ core-shell nanocomposites with highly absorbing NIR carbocyanine dyes in the outer silica shell for combined NIR imaging and photothermal therapy. Photo-thermal cell killing under $750 \mathrm{~nm}$ excitation light source demonstrated the capability of UCNPs for both diagnostic optical imaging and therapeutic thermal therapy [106]. With the development of Nano Science in the field of tumor diagnosis and treatment, the multi-modal treatment of cancers based on one kind of nanoparticles has been developed. Recently, Liu's group reported a kind of double function UCNPs. Two kinds of material were selected for the surface modification of UCNPs to achieve the dual function of PTT and PDT. Bengal Rose was used to absorb the green fluorescence of UCNP to realize the PTT, and the infrared absorption dye of IR825 was used to realize PDT [107]. The Bu team reported a MRI/NIR dual- modal nanoparticle, which can treat tumor with chemo-/ radio-/ photodynamic modals [108].

\section{Conclusions}

This article makes a review on recent progress in UCNPs for bioimaging and gastric cancer therapy. Despite that tremendous number of excellent results have been made in the past few years in this region, there are still many challenges which hinder potential applications of UCNPs as therapeutic and bioimaging agents. Firstly, the quantum yields of the most UCNPs is usually a little more than $0.005 \%$ but no more than $0.3 \%$, which significantly limits the use of these UCNPs in optical bioimaging and PDT. Secondly, the potential long-term toxicity study of rare earth doped 
UCNPs is still in its infancy. Much more systematic investigations are still in need. Thirdly, for UCNPs to be successfully used as in vivo diagnostic agents, any small changes to UCNPs shape, size, surface charge and coating will have big impacts on its behaviors toward biological systems. A systematic research on these aspects is still in great need.

\section{Acknowledgments}

This work is supported by the National Key Basic Research Program (973 Project) (No. 2015CB931802), the National Natural Scientific Foundation of China (Grant Nos. 81225010, 81028009 and 31170961), the 863 project of China (Project No. 2014AA020700) and the Shanghai Science and Technology Fund (13NM1401500).

\section{References}

[1] A. Patra, et al., Upconversion in $\mathrm{Er}^{3+}: \mathrm{ZrO}_{2}$ Nanocrystals. The Journal of Physical Chemistry B, 2002, 106(8): 19091912.

[2] A. Patra, et al., Fluorescence upconversion properties of $\mathrm{Er}^{3+}$-doped $\mathrm{TiO}_{2}$ and $\mathrm{BaTiO}_{3}$ nanocrystallites. Chemistry of Materials, 2003, 15(19): 3650-3655.

[3] X. Wang, et al., Luminescence spectroscopy and visible upconversion properties of $\mathrm{Er}^{3+}$ in $\mathrm{ZnO}$ nanocrystals. Journal of Physical Chemistry B, 2004, 108(48): 1840818413.

[4] V. Venkatramu, et al., Synthesis and luminescence properties of $\mathrm{Er}^{3+}$-doped $\mathrm{Lu}_{3} \mathrm{Ga}_{5} \mathrm{O}_{12}$ nanocrystals. Journal of Luminescence, 2008, 128(5-6): 811-813.

[5] V. Venkatramu, et al., Synthesis, structure and luminescence of $\mathrm{Er}^{3+}$-doped $\mathrm{Y}_{3} \mathrm{Ga}_{5} \mathrm{O}_{12}$ nano-garnets. $J$ Mater Chem, 2012, 22(27): 13788-13799.

[6] K.U. Kumar, et al., Multicolor upconversion emission and color tunability in $\mathrm{Tm}^{3+} / \mathrm{Er}^{3+} / \mathrm{Yb}^{3+} \mathrm{Tr}$-doped $\mathrm{NaNbO}_{3}$ nanocrystals. Materials Express, 2012, 2(4): 294-302.

[7] H. Guo, et al., Visible upconversion in rare earth iondoped $\mathrm{Gd}_{2} \mathrm{O}_{3}$ nanocrystals. Journal of Physical Chemistry B, 2004, 108(50): 19205-19209.

[8] M. Rai, et al., Infrared to visible upconversion in $\mathrm{Ho}^{3+} /$ $\mathrm{Yb}^{3+}$ co-doped $\mathrm{Y}_{2} \mathrm{O}_{3}$ phosphor: effect of laser input power and external temperature. Spectrochim Acta A Mol Biomol Spectrosc, 2012, 97: 825-829

[9] D.Y. Li, et al., White upconversion emission in $\mathrm{Yb}^{3+} /$ $\mathrm{Tm}^{3+} / \mathrm{Ho}^{3+}$ doped $\mathrm{SrMoO}_{4}$ nanocrystals by high excited state energy transfer. Journal of Alloys and Compounds, 2013, 550: 509-513.

[10] Y. Wei, et al., Polyol-mediated synthesis and luminescence of lanthanide-doped $\mathrm{NaYF}_{4}$ nanocrystal upconversion phosphors. Journal of Alloys and Compounds, 2008, 455(1-2): 376-384.

[11] F. Zhang, et al., Uniform nanostructured arrays of sodium rare-earth fluorides for highly efficient multicolor upconversion luminescence. Angewandte Chemie International Edition, 2007, 46(42): 7976-7979.

[12] J. Jin, et al., Polymer-coated $\mathrm{NaYF}_{4}: \mathrm{Yb}^{(3)(+)}, \mathrm{Er}^{(3)(+)}$ upconversion nanoparticles for charge-dependent cellular imaging. ACS Nano, 2011, 5(10): 7838-7847.

[13] D. Yang, et al., Hollow structured upconversion luminescent $\mathrm{NaYF}_{(4)}: \mathrm{Yb}^{(3)(+)}, \mathrm{Er}^{(3)(+)}$ nanospheres for cell imaging and targeted anti-cancer drug delivery. Biomaterials, 2013, 34(5): 1601-1612.

[14] J.W. Zhao, et al., A facile approach to fabrication of hexagonal-phase $\mathrm{NaYF}_{4}$ : $\mathrm{Yb}^{3+}, \mathrm{Er}^{3+}$ hollow nanospheres: Formation mechanism and upconversion luminescence. European Journal of Inorganic Chemistry, 2010(12): 1813-1819.

[15] Y.J. Liang, et al., Hydrothermal synthesis and upconversion luminescent properties of $\mathrm{YVO}_{4}: \mathrm{Yb}^{3+}, \mathrm{Er}^{3+}$ nanoparticles. Journal of Alloys and Compounds, 2013, 552: 289-293.

[16] G.S. Yi, G.M. Chow, Colloidal $\mathrm{LaF}_{3}$ : Yb,Er, $\mathrm{LaF}_{3}$ : $\mathrm{Yb}, \mathrm{Ho}$ and $\mathrm{LaF}_{3}$ : $\mathrm{Yb}, \mathrm{Tm}$ nanocrystals with multicolor upconversion fluorescence. J Mater Chem, 2005, 15(41): 4460-4464.

[17] Heer, S., et al., Highly efficient multicolour upconversion emission in transparent colloids of lanthanide-doped $\mathrm{NaYF}_{4}$ nanocrystals. Adv Mater, 2004, 16(23-24): 21022105.

[18] S. Heer, et al., Blue, green, and red upconversion emission from lanthanide-doped $\mathrm{LuPO}_{4}$ and $\mathrm{YbPO}_{4}$ nanocrystals in a transparent colloidal solution. Angewandte Chemie International Edition, 2003, 42(27): 3179-3182.

[19] G. Yi, et al., Synthesis, characterization, and biological application of size-controlled nanocrystalline $\mathrm{NaYF}_{4}: \mathrm{Yb}$, Er infrared-to-visible up-conversion phosphors. Nano Lett, 2004, 4(11): 2191-2196.

[20] Y.W. Zhang, et al., Single-crystalline and monodisperse $\mathrm{LaF}_{3}$ triangular nanoplates from a single-source precursor. J Am Chem Soc, 2005, 127(10): 3260-3261.

[21] A. Yin, et al., Colloidal synthesis and blue based multicolor upconversion emissions of size and composition controlled monodisperse hexagonal $\mathrm{NaYF}_{4}$ : Yb, Tm nanocrystals. Nanoscale, 2010, 2(6): 953-959.

[22] H.X. Mai, et al., High-quality sodium rare-earth fluoride nanocrystals: Controlled synthesis and optical properties. J Am Chem Soc, 2006, 128(19): 6426-6436.

[23] H.X. Mai, et al., Highly efficient multicolor up-conversion emissions and their mechanisms of monodisperse $\mathrm{NaYF}_{4}$ : $\mathrm{Yb}$, Er core and core/shell-structured nanocrystals. The Journal of Physical Chemistry C, 2007, 111(37): 1372113729.

[24] Q. Liu, et al., Sub-10 $\mathrm{nm}$ hexagonal lanthanidedoped $\mathrm{NaLuF}_{4}$ upconversion nanocrystals for sensitive bioimaging in vivo. J Am Chem Soc, 2011, 133(43): 17122-17125.

[25] J.C. Boyer, et al., Synthesis of colloidal upconverting $\mathrm{NaYF}_{4}$ nanocrystals doped with $\mathrm{Er}^{3+}, \mathrm{Yb}^{3+}$ and $\mathrm{Tm}^{3+}, \mathrm{Yb}^{3+}$ via thermal decomposition of lanthanide trifluoroacetate precursors. J Am Chem Soc, 2006, 128(23): 7444-7445.

[26] J.C. Boyer, L.A. Cuccia and J.A. Capobianco, synthesis of colloidal upconverting $\mathrm{NaYF}_{4}: \mathrm{Er}^{3+} / \mathrm{Yb}^{3+}$ and $\mathrm{Tm}^{3+} /$ $\mathrm{Yb}^{3+}$ monodisperse nanocrystals. Nano Lett, 2007, 7(3): $847-852$.

[27] R. Naccache, et al., Controlled synthesis and water dispersibility of hexagonal phase $\mathrm{NaGdF}_{4}: \mathrm{Ho}^{3+} / \mathrm{Yb}^{3+}$ nanoparticles. Chemistry of Materials, 2009, 21(4): 717723.

[28] F. Vetrone, V. Mahalingam and J.A. Capobianco, Nearinfrared-to-blue upconversion in colloidal $\mathrm{BaYF}_{5}: \mathrm{Tm}^{3+}$, $\mathrm{Yb}^{3+}$ nanocrystals. Chemistry of Materials, 2009, 21(9): $1847-1851$

[29] F. Vetrone, et al., The active-core/active-shell approach: A strategy to enhance the upconversion luminescence in lanthanide-doped nanoparticles. Advanced Functional Materials, 2009, 19(18): 2924-2929.

[30] W.J. Huang, et al., Uniform $\mathrm{NaYF}_{4} \mathrm{~N}$ : $\mathrm{Yb}, \mathrm{Tm}$ hexagonal submicroplates: Controlled synthesis and enhanced UV and blue upconversion luminescence. Materials Research 
Bulletin, 2013, 48(2): 300-304.

[31] W.J. Huang, et al., Controlled synthesis and upconversion luminescence properties of $\mathrm{Yb}^{3+}-\mathrm{Tm}^{3+}$ codoped $\mathrm{NaYF}_{4}$ hexagonal submicroplates. In J.C.M. Kao, M. Hou and R. Chen (Ed.), Frontier of Nanoscience and Technology. Trans Tech Publications Ltd, 2012: 117-120.

[32] Q. Liu, et al., Multifunctional rare-earth self-assembled nanosystem for tri-modal upconversion luminescence/ fluorescence/positron emission tomography imaging. Biomaterials, 2011, 32(32): 8243-8253.

[33] A.M. Pires, et al., Low-temperature upconversion spectroscopy of nanosized $\mathrm{Y}_{2} \mathrm{O}_{3}$ : Er, $\mathrm{Yb}$ phosphor. Journal of Applied Physics, 2005, 98(6): 063529-063529-7.

[34] M. He, et al., Dual phase-controlled synthesis of uniform lanthanide-doped $\mathrm{NaGdF}_{4}$ upconversion nanocrystals via an OA/Ionic liquid two-phase system for In vivo Gualmodality Imaging. Advanced Functional Materials, 2011, 21(23): 4470-4477.

[35] J. Wang, et al., One-step synthesis of highly water-soluble $\mathrm{LaF}_{3}: \mathrm{Ln}^{3+}$ nanocrystals in methanol without using any ligands. Nanotechnology, 2007, 18(46): 465606.

[36] Y. Wei, et al., Polyol-mediated synthesis of water-soluble $\mathrm{LaF}_{3}: \mathrm{Yb}, \mathrm{Er}$ upconversion fluorescent nanocrystals. Materials Letters, 2007, 61(6): 1337-1340.

[37] D. Hu, et al., A facile method to synthesize superparamagnetic and up-conversion luminescent $\mathrm{NaYF}_{4}: \mathrm{Yb}, \mathrm{Er} / \mathrm{Tm} @ \mathrm{SiO}_{2} @ \mathrm{Fe}_{3} \mathrm{O}_{4}$ nanocomposite particles and their bioapplication. J Mater Chem, 2011, 21(30): 11276-11282.

[38] H. Lu, et al., Synthesis and characterization of multifunctional nanoparticles possessing magnetic, upconversion fluorescence and bio-affinity properties. $J$ Mater Chem, 2004, 14(8): 1336-1341.

[39] J.H. Zeng, et al., Synthesis of complex rare earth fluoride nanocrystal phosphors. Nanotechnology, 2006, 17(14): 3549-3555.

[40] J.H. Zeng, et al., Synthesis and upconversion luminescence of hexagonal-phase $\mathrm{NaYF}_{4}: \mathrm{Yb}, \mathrm{Er}^{3+}$ phosphors of controlled size and morphology. Adv Mater, 2005, 17(17): 2119-2123.

[41] P. Ghosh, et al., Influence of surface coating on the upconversion emission properties of LaPO [sub 4]: $\mathrm{Yb} /$ Tm core-shell nanorods. Journal of Applied Physics, 2009, 105(11): 113532-113535.

[42] C. Li, et al., Highly uniform and monodisperse $\beta-\mathrm{NaYF}_{4}$ : $\mathrm{Ln}^{3+}(\mathrm{Ln}=\mathrm{Eu}, \mathrm{Tb}, \mathrm{Yb} / \mathrm{Er}$, and $\mathrm{Yb} / \mathrm{Tm})$ hexagonal microprism crystals: Hydrothermal synthesis and luminescent properties. Inorg Chem, 2007, 46(16): 63296337.

[43] D.K. Ma, et al., Rare-earth-ion-doped hexagonal-phase $\mathrm{NaYF}_{4}$ nanowires: controlled synthesis and luminescent properties. The Journal of Physical Chemistry C, 2009, 113(19): 8136-8142.

[44] Z.L. Wang, J.H. Hao, and H.L.W. Chan, Down- and upconversion photoluminescence, cathodoluminescence and paramagnetic properties of $\mathrm{NaGdF}_{4}: \mathrm{Yb}^{3+}, \mathrm{Er}^{3+}$ submicron disks assembled from primary nanocrystals. J Mater Chem, 2010, 20(16): 3178-3185.

[45] Zhao, J., et al., Controlled synthesis, formation mechanism, and great enhancement of red upconversion luminescence of $\mathrm{NaYF}_{4}: \mathrm{Yb}^{3+}, \mathrm{Er}^{3+}$ nanocrystals/ submicroplates at low doping level. J Phys Chem B, 2008, 112(49): 15666-15672.

[46] D. Yang, et al., One-step synthesis of small-sized and water-soluble $\mathrm{NaREF}_{(4)}$ upconversion nanoparticles for in vitro cell imaging and drug delivery. Chemistry, 2013, 19(8): 2685-2694.

[47] Sikora, B., et al., Transport of $\mathrm{NaYF}_{4}: \mathrm{Er}^{3+}, \mathrm{Yb}^{3+}$ upconverting nanoparticles into HeLa cells. Nanotechnology, 2013, 24(23): 235702.
[48] J. Yang, et al., One-step hydrothermal synthesis of carboxyl-functionalized upconversion phosphors for bioapplications. Chemistry, 2012, 18(43): 13642-13650.

[49] Z.L. Wang, et al., Simultaneous synthesis and functionalization of water-soluble up-conversion nanoparticles for in-vitro cell and nude mouse imaging. Nanoscale, 2011, 3(5): 2175-2181.

[50] Y. Gao, T.Y. Cao and F.Y. Li, Water-soluble upconversion nanophosphors with cooperative ligands for in vivo lymph node imaging. Chinese Journal of Inorganic Chemistry, 2012, 28(10): 2043-2048.

[51] M. Wang, et al., One-step synthesis and characterization of water-soluble $\mathrm{NaYF}_{4}$ : Yb, Er/Polymer nanoparticles with efficient up-conversion fluorescence. Journal of Alloys and Compounds, 2009, 485(1-2): L24-L27.

[52] Z. Wang, et al., One-pot synthesis of water-soluble and carboxyl-functionalized beta- $\mathrm{NaYF}_{4}: \mathrm{Yb}, \operatorname{Er}(\mathrm{Tm})$ upconversion nanocrystals and their application for bioimaging. J Mater Chem, 2012, 22(24): 12186-12192.

[53] T. Cao, et al., High-quality water-soluble and surfacefunctionalized upconversion nanocrystals as luminescent probes for bioimaging. Biomaterials, 2011, 32(11): 29592968.

[54] C. Chen, et al., Ionic liquid-based route to spherical $\mathrm{NaYF}_{4}$ nanoclusters with the assistance of microwave radiation and their multicolor upconversion luminescence. Langmuir, 2010, 26(11): 8797-803.

[55] X. Liu, et al., Ionothermal synthesis of hexagonal-phase $\mathrm{NaYF}_{(4)}: \mathrm{Yb}^{(3+)}, \mathrm{Er}^{\left(3^{++}\right)} / \mathrm{Tm}^{(3+)}$ upconversion nanophosphors. Chem Commun (Camb), 2009: 6628-6630.

[56] M. He, et al., Phase- and size-controllable synthesis of hexagonal upconversion rare-earth fluoride nanocrystals through an oleic acid/ionic liquid two-phase system. Chemistry-a European Journal, 2012, 18(19): 5954-5969.

[57] J.C. Boyer, et al., Surface modification of upconverting $\mathrm{NaYF}_{4}$ nanoparticles with PEG-phosphate ligands for NIR $(800 \mathrm{~nm})$ biolabeling within the biological window. Langmuir, 2009, 26(2): 1157-1164.

[58] M. Wang, et al., Immunolabeling and NIR-excited fluorescent imaging of HeLa cells by using $\mathrm{NaYF}_{4}: \mathrm{Yb}, \mathrm{Er}$ upconversion nanoparticles. ACS Nano, 2009, 3(6): 15801586.

[59] M. Deng, et al., Monodisperse upconversion $\mathrm{NaYF}_{4}$ nanocrystals: Syntheses and bioapplications. Nano Research, 2011, 4(7): 685-694.

[60] C. Salthouse, et al., Design and demonstration of a smallanimal up-conversion imager. Opt Express, 2008, 16(26): 21731-21737.

[61] H. Kobayashi, et al., In vivo multiple color lymphatic imaging using upconverting nanocrystals. J Mater Chem, 2009, 19(36): 6481-6484.

[62] L. Cheng, et al., Highly-sensitive multiplexed in vivo imaging using pegylated upconversion nanoparticles. Nano Research, 2010, 3(10): 722-732.

[63] Q. Chen, et al., Functionalization of upconverted luminescent $\mathrm{NaYF}_{4}$ : $\mathrm{Yb} / \mathrm{Er}$ nanocrystals by folic acidchitosan conjugates for targeted lung cancer cell imaging. J Mater Chem, 2011, 21(21): 7661-7667.

[64] L.L. Li, et al., Biomimetic surface engineering of lanthanide-doped upconversion nanoparticles as versatile bioprobes. Angew Chem Int Ed Engl, 2012, 51(25): 61216125.

[65] L.Q. Xiong, et al., Synthesis, characterization, and in vivo targeted imaging of amine-functionalized rare-earth upconverting nanophosphors. Biomaterials, 2009, 30(29): $5592-5600$.

[66] N.M. Idris, et al., Tracking transplanted cells in live animal using upconversion fluorescent nanoparticles. Biomaterials, 2009, 30(28): 5104-5113.

[67] D. Cui, et al., Fluorescent magnetic nanoprobes for in vivo 
targeted imaging and hyperthermia therapy of prostate cancer. Nano Biomed. Eng, 2009, 1(1): 61-74.

[68] T. Zako, et al., Cyclic RGD peptide-labeled upconversion nanophosphors for tumor cell-targeted imaging. Biochem Biophys Res Commun, 2009, 381(1): 54-58.

[69] L.Y. Pan, et al., Phase and size controllable synthesis of $\mathrm{NaYbF}_{4}$ nanocrystals in oleic acid/ionic liquid two-phase system for targeted fluorescent imaging of gastric cancer. Theranostics, 2013, 3(3): 210-222.

[70] R. Kumar, et al., Combined optical and MR bioimaging using rare earth ion doped $\mathrm{NaYF}_{4}$ nanocrystals. Advanced Functional Materials, 2009, 19(6): 853-859.

[71] Z. Liu, et al., Long-circulating $\mathrm{Er}^{3+}$-doped $\mathrm{Yb}_{2} \mathrm{O}_{3}$ upconversion nanoparticle as an in vivo X-Ray $\mathrm{CT}$ imaging contrast agent. Biomaterials, 2012, 33(28): 6748-6757.

[72] Ma, J., et al., Folic acid-conjugated $\mathrm{LaF}_{3}$ : Yb,Tm@ $\mathrm{SiO}_{2}$ nanoprobes for targeting dual-modality imaging of upconversion luminescence and X-ray computed tomography. J Phys Chem B, 2012, 116(48): 1406214070 .

[73] H. Xing, et al., Multifunctional nanoprobes for upconversion fluorescence, MR and CT trimodal imaging. Biomaterials, 2012, 33(4): 1079-1089.

[74] S. Zeng, et al., PEG modified $\mathrm{BaGdF}_{(5)}: \mathrm{Yb} /$ Er nanoprobes for multi-modal upconversion fluorescent, in vivo X-ray computed tomography and biomagnetic imaging. Biomaterials, 2012, 33(36): 9232-9238.

[75] Q. Xiao, et al., Radiopaque fluorescence-transparent TaOx decorated upconversion nanophosphors for in vivo $\mathrm{CT} /$ MR/UCL trimodal imaging. Biomaterials, 2012. 33(30): 7530-7539.

[76] H.Y. Xing, et al., $\mathrm{A} \mathrm{NaYbF}_{4}: \mathrm{Tm}^{3+}$ nanoprobe for $\mathrm{CT}$ and NIR-to-NIR fluorescent bimodal imaging. Biomaterials, 2012, 33(21): 5384-5393.

[77] G. Zhang, et al., Dual modal in vivo imaging using upconversion luminescence and enhanced computed tomography properties. Nanoscale, 2011, 3(10): 43654371.

[78] G. Gao, et al., One-pot hydrothermal synthesis of lanthanide ions doped one-dimensional upconversion submicrocrystals and their potential application in vivo CT imaging. Nanoscale, 2013, 5(1): 351-362.

[79] J. Zhou, et al., Water-stable $\mathrm{NaLuF}_{4}$-based upconversion nanophosphors with long-term validity for multimodal lymphatic imaging. Biomaterials, 2012, 33(26): 62016210.

[80] F.Y. Liu, et al., Conjugation of $\mathrm{NaGdF}_{4}$ upconverting nanoparticles on silica nanospheres as contrast agents for multi-modality imaging. Biomaterials, 2013, 34(21): 5218-5225.

[81] A. Xia, et al., $\mathrm{Gd}^{3+}$ complex-modified $\mathrm{NaLuF}_{4}$-based upconversion nanophosphors for trimodality imaging of NIR-to-NIR upconversion luminescence, X-Ray computed tomography and magnetic resonance. Biomaterials, 2012, 33(21): 5394-5405.

[82] F. Liu, et al., Facile preparation of doxorubicinloaded upconversion@polydopamine nanoplatforms for simultaneous in vivo multimodality imaging and chemophotothermal synergistic therapy. Adv Healthc Mater, 2015, 4(4): 559-568.

[83] C. Xie, et al., Preparation of a novel amino functionalized fluorescein-doped silica nanoparticle for $\mathrm{pH}$ probe. Nano Biomed. Eng, 2009, 1(1): 27-31.

[84] D. Liu, et al., The Three-dimensional images and intracellular calcium analysis of weigela floridacv and lonicera japonica thunb pollen. Nano Biomed. Eng, 2009, 1(1): 57-60.

[85] H. Yang, et al. Detection of nanobubbles at the interface of $\mathrm{TiO}_{2}$ coated mica in water. Nano Biomed. Eng, 2009, 1(1): $75-79$.
[86] C. He, et al., Physical-chemical properties and in vitro biocompatibility assessment of spider silk, collagen and polyurethane nanofiber scaffolds for vascular tissue engineering. Nano Biomed. Eng, 2009, 1(1): 80-88.

[87] C. Wang, et al., Towards whole-body imaging at the single cell level using ultra-sensitive stem cell labeling with oligo-arginine modified upconversion nanoparticles. Biomaterials, 2012, 33(19): 4872-4881.

[88] R. Deng, et al., Intracellular glutathione detection using $\mathrm{MnO}_{2}$-nanosheet-modified upconversion nanoparticles. $J$ Am Chem Soc, 2011, 133(50): 20168-20171.

[89] C. Wang, et al., Drug delivery with upconversion nanoparticles for multi-functional targeted cancer cell imaging and therapy. Biomaterials, 2011, 32(4): 11101120 .

[90] J.N. Liu., et al., Simultaneous nuclear imaging and intranuclear drug delivery by nuclear-targeted multifunctional upconversion nanoprobes. Biomaterials, 2012, 33(29): 7282-7290.

[91] G. Tian, et al., $\mathrm{Mn}^{2+}$ dopant-controlled synthesis of $\mathrm{NaYF}_{4}: \mathrm{Yb} / \mathrm{Er}$ upconversion nanoparticles for in vivo imaging and drug delivery. Adv Mater, 2012, 24(9): 12261231.

[92] H. Guo, et al., Upconversion nanoparticles modified with aminosilanes as carriers of DNA vaccine for foot-andmouth disease. Appl Microbiol Biotechnol, 2012, 95(5): 1253-1263.

[93] F. Zhang, et al., Mesoporous multifunctional upconversion luminescent and magnetic "nanorattle" materials for targeted chemotherapy. Nano Lett, 2012, 12(1): 61-67.

[94] P.Y. Qiu, et al., An anion-induced hydrothermal oriented-explosive strategy for the synthesis of porous upconversion nanocrystals. Theranostics, 2015, 5(5): 456468.

[95] J. Shan, et al., NIR-to-visible upconversion nanoparticles for fluorescent labeling and targeted delivery of siRNA. Nanotechnology, 2009, 20(15): 155101.

[96] Y. Yang, et al., NIR light controlled photorelease of siRNA and its targeted intracellular delivery based on upconversion nanoparticles. Nanoscale, 2013, 5(1): 231238.

[97] Y.E. Koo, et al., Photonic explorers based on multifunctional nanoplatforms for biosensing and photodynamic therapy. Appl Opt, 2007, 46(10): 19241930.

[98] Y.E. Lee, and R. Kopelman, Polymeric nanoparticles for photodynamic therapy. Methods Mol Biol, 2011, 726: 151-178.

[99] G.R. Reddy, et al., Vascular targeted nanoparticles for imaging and treatment of brain tumors. Clin Cancer Res, 2006, 12(22): 6677-6686.

[100] Gupta, et al., Multifunctional nanoplatforms for fluorescence imaging and photodynamic therapy developed by post-loading photosensitizer and fluorophore to polyacrylamide nanoparticles. Nanomedicine, 2012, 8(6): 941-950.

[101] S. Wang, et al., Novel methods to incorporate photosensitizers into nanocarriers for cancer treatment by photodynamic therapy. Lasers Surg Med, 2011, 43(7): 686-695.

[102] X. Huang, et al., Cancer cell imaging and photothermal therapy in the near-infrared region by using gold nanorods. J Am Chem Soc, 2006, 128(6): 2115-2120.

[103] B. Dong, et al., Multifunctional $\mathrm{NaYF}_{4}: \mathrm{Yb}^{3+}, \mathrm{Er}^{3+} @ \mathrm{Ag}$ core/shell nanocomposites: integration of upconversion imaging and photothermal therapy. J Mater Chem, 2011, 21(17): 6193-6200.

[104] L. Cheng, et al., Facile preparation of multifunctional upconversion nanoprobes for multimodal imaging and dual-targeted photothermal therapy. Angew Chem Int Ed 
Engl, 2011, 50(32): 7385-7390.

[105] L. Cheng, et al., Multifunctional nanoparticles for upconversion luminescence/MR multimodal imaging and magnetically targeted photothermal therapy. Biomaterials, 2012, 33(7): 2215-2222.

[106] G.B. Shan, R. Weissleder and S.A. Hilderbrand, Upconverting organic dye doped core-shell nanocomposites for dual-modality NIR imaging and photothermal therapy. Theranostics, 2013, 3(4): 267-274.

[107] Q. Chen, et al., Protein modified upconversion nanoparticles for imaging-guided combined photothermal and photodynamic therapy. Biomaterials, 2014, 35(9):
2915-2923.

[108] W. Fan, et al., A smart upconversion-based mesoporous silica nanotheranostic system for synergetic chemo-/ radio-/photodynamic therapy and simultaneous MR/UCL imaging. Biomaterials, 2014, 35(32): 8992-9002.

Copyright $(2016$ Yuming Yang, Yu Han and Caixia Yue. This is an open-access article distributed under the terms of the Creative Commons Attribution License, which permits unrestricted use, distribution, and reproduction in any medium, provided the original author and source are credited. 